\title{
Local Area Networks for Radiology
}

\author{
Samuel J. Dwyer, III, Nicholas J. Mankovich, Glendon G. Cox, and Roger A. Bauman
}

\begin{abstract}
This article is a tutorial on local area networks (LAN) for radiology applications. LANs are being implemented in radiology departments for the management of text and images, replacing the inflexible point-to-point wiring between two devices (computer-to-terminal). These networks enable the sharing of computers and computer devices, reduce equipment costs, and provide improved reliability. Any LAN must include items from the following four categories: transmission medium, topology, data transmission mode, and access protocol. Media for local area networks are twisted pair, coaxial, and optical fiber cables. The topology of these networks include the star, ring, bus, tree, and circuit-switching. Data transmission modes are either analog signals or digital signals. Access protocol methods include the broadcast bus system and the ring system. A performance measurement for a LAN is the throughput rate as a function of the number of active computer nodes. Standards for LANs help to ensure that products purchased from multiple manufacturers will operate successfully.

(1) 1988 by W.B. Saunders Company.
\end{abstract}

KEY WORDS: Local area network (LAN), transmission medium, topology, data transmission mode, acess protocols, throughput, and standards.

$\mathbf{I}^{\mathrm{N}}$ NHE EARLY 1950s, central computers were connected directly (point-to-point) to terminals, printers, and other computer peripheral devices. The number of peripheral devices and communication links increased significantly in the early 1960s with the introduction of timesharing computers. Time-sharing allowed a larger number of users at remote sites to satisfy their computer needs without having to travel to the computer facility. As these remote devices increased in number, the cost of point-to-point links became large.

Two significant developments reduced the cost of these point-to-point communication links. First, a number of remote peripherals in a small

From the Department of Diagnostic Radiology, University of Kansas Medical Center, Kansas City; and the Department of Radiological Sciences, UCLA School of Medicine, Los Angeles and the Department of Radiology.

Address reprint requests to Samuel J. Dwyer, III, PhD, Department of Diagnostic Radiology, The University of Kansas Medical Center, Kansas City, KS 66103.

(c) 1988 by W.B. Saunders Company.

0897-1889/88/0101-0004\$03.00/0 area were connected to a concentrator which was connected by a single communication link to the central computer. This reduced the cost of data communication links by replacing a number of them with a single link. Second, the computer was relieved of all communication processing tasks by special processors called front ends.

In the early 1970 s, large area data networks became available for connecting multiple computer systems and peripheral devices. These networks became known as wide area networks. The cost of communication links in these wide area networks remains a significant percentage of the total operating cost.

In the 1980s, solid state technology advances have provided the powerful microcomputer systems and inexpensive data communication devices of today. ${ }^{1-4}$ These developments have permitted movement away from large time-sharing computers to distributed processing and resource sharing via inexpensive data networks. By encoding data into small packets, it can be transmitted over a network and reassembled at the receiving site. In the middle 1980s, single owner, small area data networks were developed. These small area data networks are called local area networks (LANs).$^{5-8}$

Using either a wide area network (many miles) or a LAN (several miles), one is able to access any computer or computer device on the network (assuming necessary security approvals). Personal computers can act as a computer or as a terminal over a network. The networks are combinations of hardware and software dedicated to markedly more flexible access to and from sharing of resources. ${ }^{9-11}$ The possibility of electronic mail over the entire network is an added benefit.

This tutorial is intended to provide an introduction to the basics of LANs. These LANs are already in place in many small and large radiology departments. They are used to link two or more personal computers to each other and to computer peripheral devices in office automation and billing systems. The terminals and other devices on radiology and hospital information systems are often connected via a LAN. LANs also move images between devices in picture 
archiving and communication systems. No attempt will be made to provide details of those applications other than a few diagrams.

The focus of this presentation is to identify and introduce the components of a LAN and how they work together. These LAN components are the transmission medium, the topology, the data transmission mode, the access protocol, and the network nodes (computers and other devices). Because there are many possible ways to combine components from these categories, it is virtually impossible to simply name a LAN without many qualifiers (exception: a commercial product name). Talking about a fiberoptic LAN gives information only about the transmission medium, a very incomplete description. Teasing apart the various component parts of LANs will help to remove some of the mystery that the many terms tend to create.

The transmission medium of a LAN is the physical medium used for the interconnection. The electrical signaling rate on a LAN over various transmission media ranges from 1 to 50 megabits per second (Mbps). The actual throughput of data transferred between two connected devices varies from 50 kilobits per second (Kbps) to $6 \mathrm{Mbps}$. This disparity between signaling rates and throughput data rates is due to the overhead necessary to operate a LAN. The topology of a LAN is the connection arrangement of the computers and computer devices. The access control method is the user communication protocol for transferring data on the LAN. Every connected device can communicate with every other device. Protocols are the sets of rules and conventions governing communication on a network. These protocols minimize the collisions that may occur when two or more users attempt to transfer data simultaneously.

Historically, digital communication technology for a LAN began with the packet-switching ALOHA radio network in Hawaii. ${ }^{12,13}$ The ALOHA network was established to provide access for a large number of terminals connected to a central computing facility. Packets of data were broadcast on a radio channel and received by the central computer installation. Ethernet was subsequently developed replacing the radio channel by coaxial cable. ${ }^{14-16}$ Many subsequent LAN designs have provided significant improvements. Increased demand for LANs includes widespread applications for interconnecting large computers, workstations, and business equipment in an office in the same building, microcomputers and digital devices in the same building or between buildings, and image management networks.

Radiology departments are already using personal computers, file servers, printers, and LANs for a number of office automation functions. Figure 1 illustrates a LAN in a professional fee patient-billing system which manages patient billing, insurance claims, on-line history, and transaction files. Furthermore, it acquires patients' demographic data from the Hospital Information Management System, transmits third-party payment requests, and generates revenue reports. The computer nodes are micro-
Fig 1. A LAN for a professional for patient billing system.

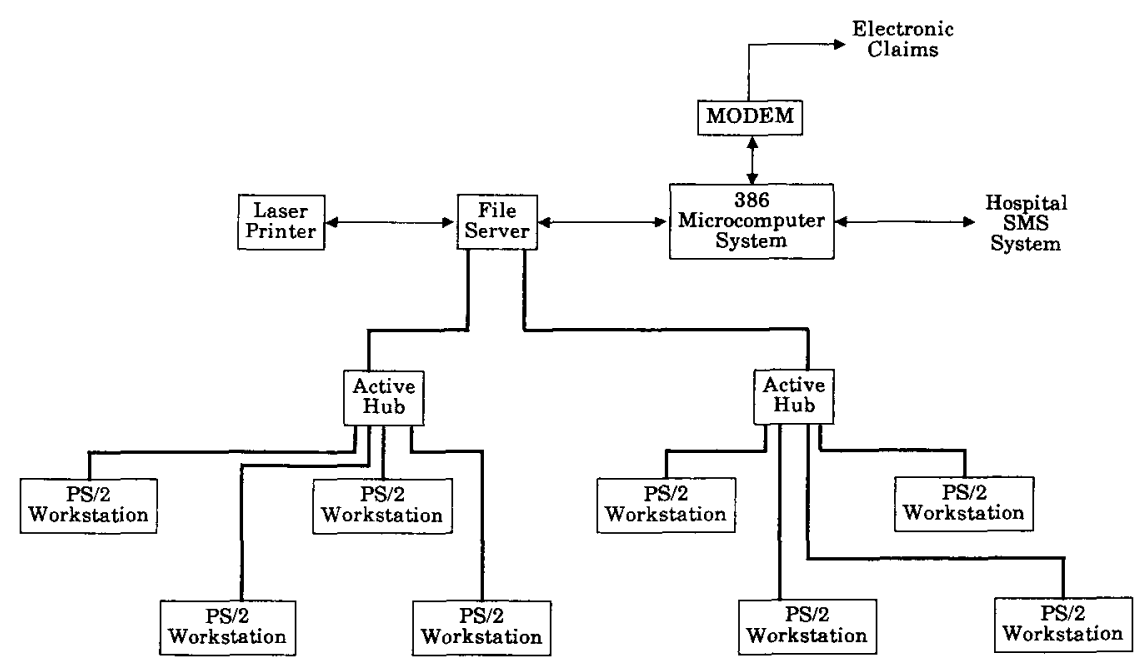




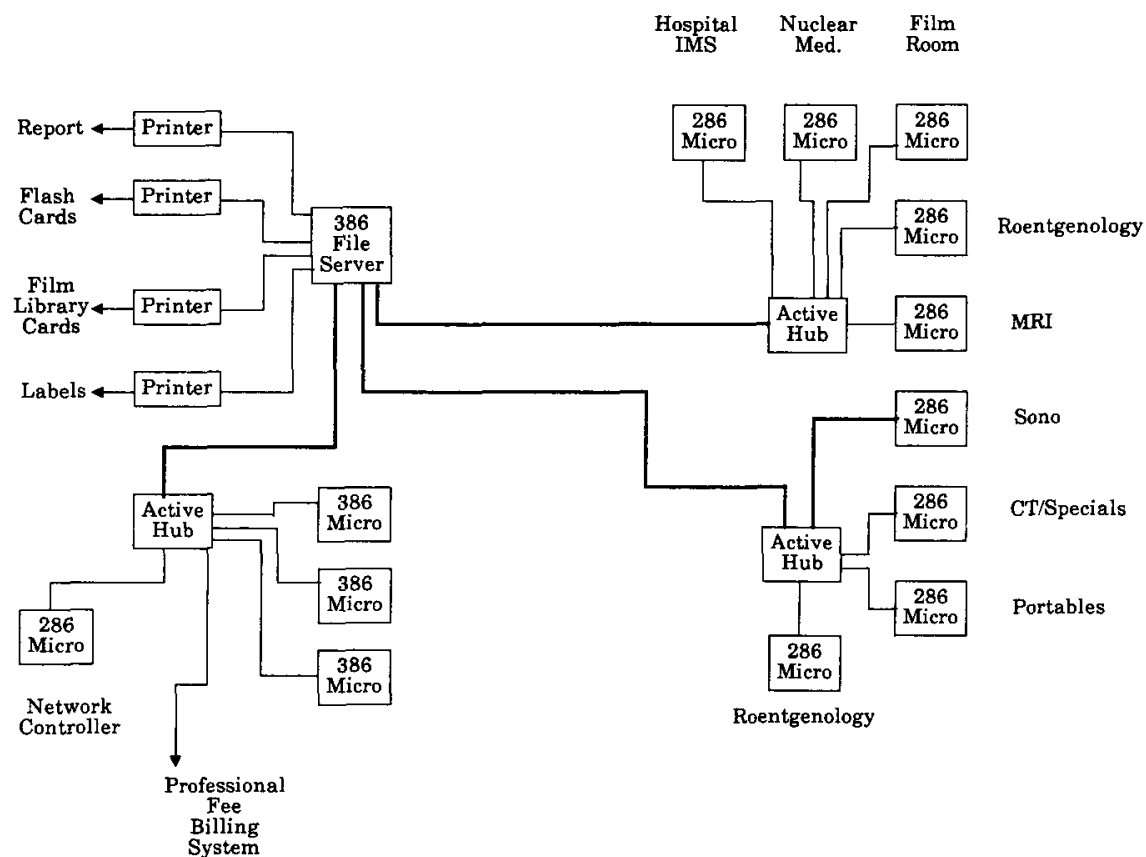

Fig 2. A LAN for a radiology information management system. computer workstation systems, a file server microcomputer, a microcomputer for running the management programs, and a laser printer (15 pages per minute) for printing patient bills, claim forms, and reports. The LAN is a 2.5 Mbps token-passing network using a distributed star topology. This professional fee billing system processes 450 patients per day, or an average of 625 examinations per day.

Figure 2 illustrates a LAN for a radiology information management system that can provide information about patient scheduling, flash card generation, film file room cards, management reports, and can track the patient throughout the department. The computer nodes are microcomputer workstations, a microcomputer file server, and printers. The LAN is a $2.5 \mathrm{Mbps}$ token passing token passing network with a distributed star topology which interfaces to the professional fee billing system and to a hospital information system.

Figure 3 illustrates a LAN for a radiology image management system. An image management system acquires digitally formatted imaging data from the imaging modalities. Digital imaging data are transmitted throughout the network. ${ }^{17,18}$ Interactive diagnosis display stations provide for the display and manipulation of the data. Hardcopy nodes generate analog film or paper recordings of the image data. Archiving nodes provide for the on-line, high access rate storage and retrieval of the digital data. Longterm archiving nodes provide medium access rate storage and retrieval of digital images. Interfaces to the department and hospital information systems are required.

\section{TRANSMISSION MEDIUM FOR A LAN}

Commonly used transmission media for LANs are twisted pair, coaxial, and optical fiber cables. ${ }^{19,20}$ A twisted pair consists of two insulated wires arranged in a regular spiral pattern. The wires are copper, which provides electrical conductivity, or steel coated with copper. Twisting of the wire pairs minimizes electromagnetic interference. Twisted pairs are widely used for analog telephone service in "local loops" that connect all phones in a limited geographic area to a central exchange. When twisted pairs are used for digital data transmission, signaling rates of 4 Mbps are attainable. Important advantages of twisted pair are easy installation and low cost. A disadvantage of a twisted pair is its susceptibility to interference and noise because it easily couples with outside electromagnetic fields.

A coaxial cable consists of two conductors; a hollow outer cylindrical conductor surrounds a single inner wire conductor. The inner and outer conductors can be either solid or stranded. The inner conductor is held in place by either regu- 
Fig 3. A LAN for a radiology image management system.

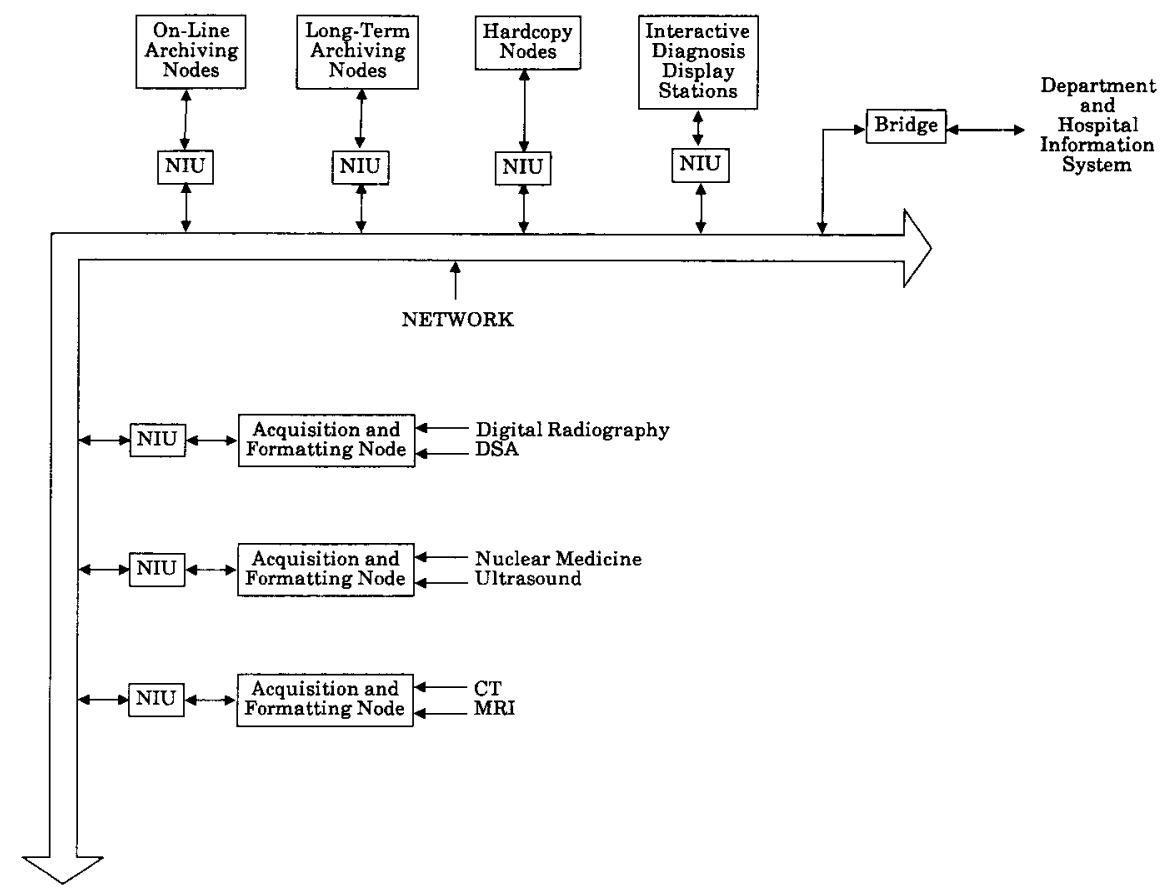

larly spaced insulating rings or a solid dielectric material. The outer conductor is covered with a jacket or shield. A single coaxial cable has a diameter of 0.4 to 1.0 inch. A $50-\Omega$ characteristic impedance coaxial cable is used for digital transmission. ${ }^{21,22}$ A $75-\Omega$ characteristic impedance coaxial cable is used for analog and digital signaling. These cables are used for community antenna television (CATV). ${ }^{23}$

An optical fiber is a thin ( 2 to $125 \mu \mathrm{m}$ ), flexible medium capable of conducting light. ${ }^{24}$ Various glasses and plastics have been used to make optical fibers. Minimal losses have been sustained using fibers of ultrapure fused silica. An optical fiber cable has a cylindrical shape and consists of three concentric sections. The core is the innermost section and consists of one or more very thin strands of fibers made of glass or plastic. The cladding is a coating surrounding each fiber whose optical properties differ from those of the core. The jacket is an outer layer which protects against moisture, crushing, and other damage. Fiber optic cables are usually packaged in groups of two or more to make up a multi-fiber cable.

Two types of light sources are used in fiber optic systems, namely, a light-emitting diode, which is a solid state device that emits light when a current is applied, or an injection laser diode, which is a solid state device that produces a narrow light beam. ${ }^{25}$ Two types of photodiode light detectors are used at the receiving end to convert the light into electrical signals, namely, a player intrinsic layer-N layer photodiode detector, which has a segment of intrinsic silicon between the $\mathrm{P}$ and $\mathrm{N}$ layers of a junction diode, or an avalanche photodiode detector, which is an avalanche solid state photodiode device. ${ }^{26}$ The chief advantage of the optical fiber cable is that it is not affected by electromagnetic interference or noise. Fiber optic links are capable of providing light transmission over 6 to $8 \mathrm{~km}$ without repeaters. They can carry signals at rates of 10 to $256 \mathrm{Mbps}$. Optical fibers require precision alignment in connecting two cables, thus splicing and tapping are relatively expensive.

The choice of a transmission medium for a LAN depends upon its applications. For textdata systems (Figs 1 and 2), twisted pair cables are excellent choices. Signaling rates of 1 to 4 Mbps provide adequate bandwidth to support tens of terminals. In addition, twisted pair cables are inexpensive to reroute. Coaxial cables are the choice when supporting signal rates of 10 to 50 Mbps. Their bandwidth is capable of supporting hundreds of terminals. They can be used for digital, analog, or mixed signaling formats. However, coaxial cables are expensive and difficult to 
install. A number of commercial LAN systems now use coaxial cables. Optical fiber cables provide the highest bandwidth for signaling, ranging from 10 to $256 \mathrm{Mbps}$. The increasing use of optical fiber cables into LANs makes this medium the future choice for radiology image management applications.

\section{TOPOLOGY OF A LAN}

The topology of a LAN is the arrangement of the computers and computer devices and the interconnections among them. ${ }^{3,5,6}$ The importance of the topology of a LAN is illustrated by determining the number of links needed to interconnect $\mathrm{N}$ devices fully. There will be $\mathrm{N}(\mathrm{N}-1) / 2$ links required to interconnect each device to all of the other devices. For example, if we were to interconnect ten devices $(\mathbf{N}=10)$ so that each device was linked to all the other devices, then a maximum of 45 links would be required. Hence, the cost of interconnection in a LAN depends significantly upon the choice of topology of the network. The topologies used in a LAN are the following: star, ring, bus (baseband, broadband), tree, and circuit switched. These arrangements connect all nodes with a varying number of links but in each case, it is less than the maximum number of links.

A star LAN topology is illustrated by Fig 4A. Communication to any connected device is controlled by the hub. Device "W" wishing to transmit to device " $X$ " sends such a request to the hub. The hub inserts this request into a service queue. When the queue services this request, devices $\mathrm{X}$ and $\mathrm{W}$ then transmit data according to their communication protocols. No interference among other users occurs since the hub establishes the data paths and blocks other paths until the X-to-W communication is complete. The advantages are that addressing each computer node is easy, all computer nodes are centrally controlled by the hub, and it is easy to detect faults. The disadvantages include vulnerability to hub failures, data throughput rates that are limited by hub speed, and the high cost of the centralized hub. Future LAN topology will move towards the star topology as the link bandwidths increase, hubs decrease in price, and programmable link data rates are implemented.

A ring topology is illustrated by Fig 4B. Communication from device $\mathrm{W}$ to device $\mathrm{X}$ is accom-
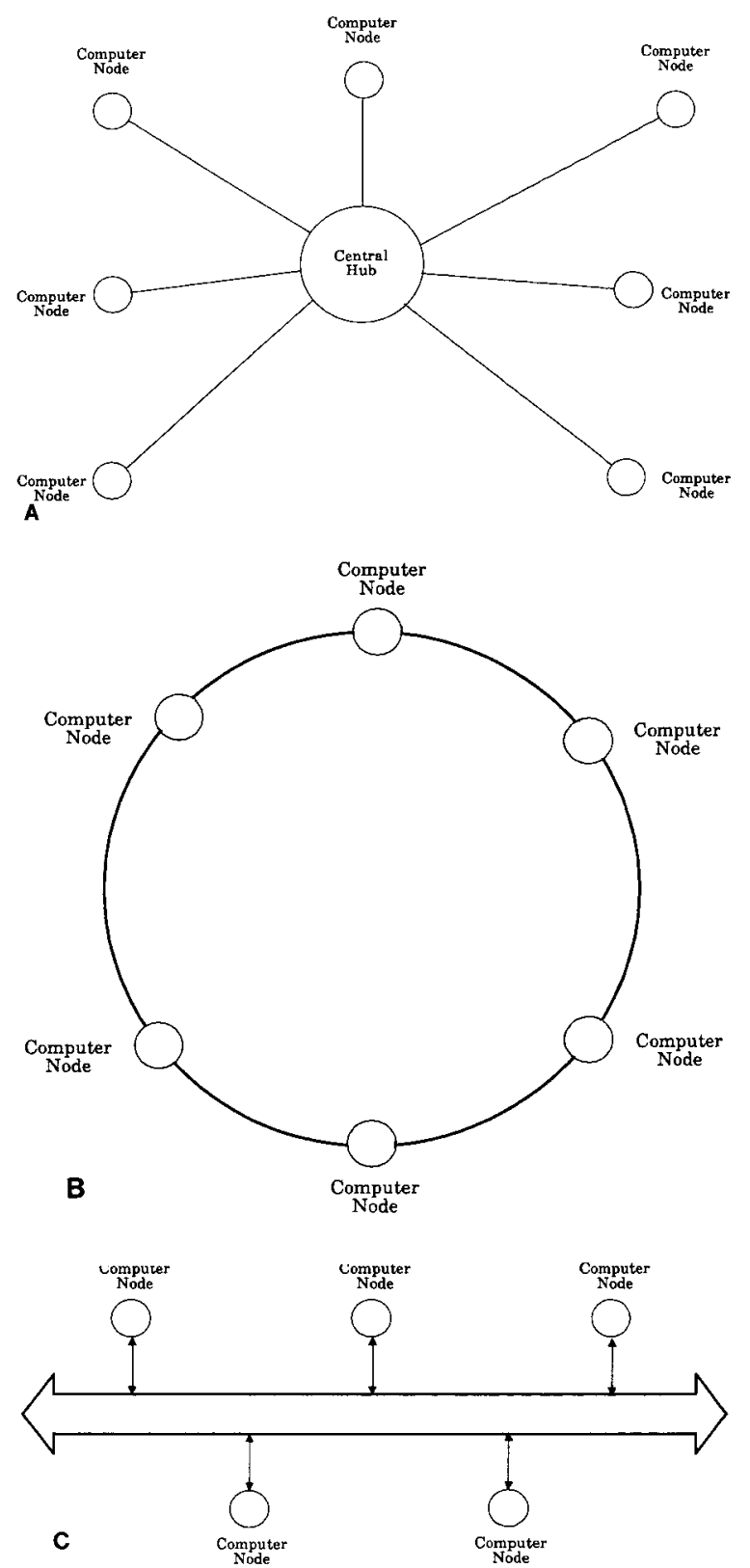

Fig 4. (A) A LAN arranged in a star topology. (B) A LAN arranged in a ring topology. (C) A LAN arranged in a bus topology.

plished by device $\mathrm{W}$ inserting its data onto the ring. The ring transmits this data from ring access point to ring access point. Each ring access point reads the destination address of the data packet. If the address is correct, then the data are read into the node and removed from the ring. If the address is not correct, then the ring access point transmits the data packet to the next ring 
access point. The advantages are: data transmission capacity is shared uniformly among all users; there is no dependence on a central device; high data transmission rates are possible; access by the user is guaranteed, even when the ring is heavily loaded; and it is inexpensive to implement. Disadvantages include: breakage of any link halts all data transmission; cable installation and routing can be difficult; modification or rerouting of the ring is difficult; each repeater at every computer node or device imposes some signal delay; and a ring network monitoring device is usually needed to maintain smooth operation of the LAN.

Figure $4 \mathrm{C}$ illustrates a LAN using bus topology. Communication on a bus topology depends upon all nodes listening to all signals on the bus. When device $\mathrm{W}$ wants to transmit to device $\mathrm{X}$, it begins transmitting its data. Device $\mathrm{W}$ listens to the bus during its own transmission. If it hears itself transmitting accurately, then it assumes the message was correctly received (assuming device $X$ is not asleep). If the signal it hears is not the same as the one being transmitted, then device $\mathrm{W}$ assumes a collision has occurred, ie, someone else is also attempting to transmit on the same bus. The advantages of bus topology are: it is easy to add additional nodes; the bus is passive, thereby improving reliability; coaxial cable is used (either $50 \Omega$ or $75 \Omega$ ); and this configuration is widely available. Disadvantages include: the access communications protocol to transmit data is complex; network interface units require buffer storage in order to achieve high data throughput rates using small packet sizes; and data throughput declines with heavy loads.

A LAN using a tree topology consists of a very high data rate baseband bus ( $256 \mathrm{Mbps}$ ), called a backbone, with multiple drop-off baseband busses (10 to $50 \mathrm{Mbps}$ ). This type of LAN topology is suitable for department-wide image management networks, and it is capable of combining fiber optics, coaxial cables, and twisted wire pairs. Because of its costly backbone link, a tree topology LAN is expensive to implement. Communication protocols are complex and require a communication controller. However, a tree topology is the likely result of the growth and integration of many small LANs into a single network.

An alternative to the packet-switched type
LAN described is a LAN based upon high-speed circuit-switching devices similar in operation to those of the telephone switched systems. ${ }^{6,27} \mathrm{~A}$ circuit-switched LAN provides interconnections among devices by switching the sending device to the receiving device. These switching devices are configured in a star topology using twisted pair wires to connect end points to the switch. Data rates to individual stations are low (64 kbps), but the bandwidth is guaranteed once a connection is made. One form of circuit-switched LAN is the digital private branch exchange. The availability of a high-speed circuit-switching device would provide LAN capabilities without complex medium access protocols.

\section{DATA TRANSMISSION MODES}

Data are defined as those entities that convey meaning, content, or interpretation of the data. ${ }^{28}$ Analog data take on continuous values over some interval, eg, voice or video. Digital data take on discrete values over some interval, eg, integers or text. A channel is a medium such as a wire, a coaxial cable, or an optical fiber. The bandwidth of a channel is the range of frequencies that it can transmit with reasonable fidelity. For example, a telephone channel bandwidth is 300 to $3400 \mathrm{~Hz}$, while voice data are in the range of 20 $\mathrm{Hz}$ to $20,000 \mathrm{~Hz}$. The limitations of channel bandwidth determine the signaling rates and data throughput rates.

The distortion of the input waveform to any communication link is caused by amplitudefrequency distortion, which is the result of nonuniform attenuation of the frequency spectrum of the transmitted signal, or phase-frequency distortion, which is the most limiting impairment to data transmission and is caused by a nonlinear phase shift of the frequency spectrum of the transmitted signal. ${ }^{21}$ Distortion is also caused by nonlinearities in data channels and low signalto-noise ratios. ${ }^{21,22}$ The small distances covered by a LAN result in small amplitude-frequency distortion, small phase-frequency distortion, and large bandwidths. Small distances and careful attention to shielding result in high signal-tonoise $(S / N)$ ratios. The relationship between bandwidth and $\mathrm{S} / \mathrm{N}$ is given by the Shannon Channel Capacity Theorem, ${ }^{22}$ ie, $\mathrm{C}=\mathrm{B} \log _{2}$ $(1+\mathrm{S} / \mathrm{N})$ where $\mathrm{C}$ is the maximum channel capacity in bits per second, $\mathrm{B}$ is the bandwidth of 
the channel (hertz), and (N) the mean square noise of the channel, which is additive and white gaussian distributed.

Data are encoded for transmission on a channel into either analog signals or digital signals. The basis for analog signaling is a continuous constant-frequency signal known as the carrier signal. Digital data are encoded by modulating one of the three characteristics of the carrier signal, ie, amplitude (amplitude-shift keying); frequency (frequency-shift keying); or phase (phase-shift keying). Analog signaling is used for lower signaling rates such as $1,200 \mathrm{bps}$ or 19.5 kbps. The basis for digital signals is the use of two different voltage levels for the two binary digits. One voltage level is used to represent a "zero," while the other voltage level represents a "one." The Manchester Code is most often used in a baseband LAN., ${ }^{5,6}$ This code is a selfclocking code in which a signal transition occurs during each data bit to enable synchronization of the hardware. The bit is a zero if a low-high transition occurs within the bit time interval and a one if a high-low transition occurs.

It is desirable to use the LAN medium efficiently by having it carry multiple signals simultaneously. This is called multiplexing. The three methods of multiplexing are: (1) time division multiplexing (TDM), also called baseband signaling, in which each device is allocated a unique time slot; (2) statistical TDM in which time slots are allocated to devices when needed; and (3) FDM, also called broadband signaling, in which each device is allocated a unique band or range of frequencies.

An example of the TDM is the pulse code modulated $\mathrm{T} 1$ carrier system. In the $\mathrm{T} 1$ carrier system, data are taken at each source and digitized to seven bits, then an eighth bit is added for signaling control. Twenty-four sources are multiplexed so that each frame is 192 bits wide $(8 \times 24)$. Data are taken at each source 8,000 times per second. The data rate of a $\mathrm{T} 1$ carrier system is $1.544 \mathrm{Mbps}([192+1$ frame bit] $\times 8,000$ ).

Baseband techniques have been widely adopted by LAN designers because the signal can be transmitted at high rates and no modulation technique is required. The advantages are that it is inexpensive to implement, the technology is simple, and it is easy to install. Disadvan- tages are that only a single channel is available, only limited distances may be covered, and capacity for high speed transmission is limited.

Broadband systems modulate information on an analog carrier wave. When applied to a LAN, the term broadband means the use of FDM and coaxial cable on which analog signaling is used. The advantages of broadband include: high data transmission capacity, large area coverage, the use of mature CATV technology, and the ability to mix data traffic types such as voice, FAX, video images, or data. The disadvantages include: modulation equipment is expensive, installation and maintenance is costly, and the propagation delay is twice that of baseband systems. Another example of FDM is the analog voice telephone system. A band width of $4 \mathrm{kHz}$ is allocated for each voice channel.

\section{ACCESS CONTROL METHODS}

The responsibility for allocating the LAN capacity is usually distributed among all the nodes on the network. ${ }^{27,29}$ No single node is used as an access controller. The common LAN access control methods are the broadcast-bus system and the ring system. ${ }^{5,6}$

A broadcast system is one in which a message, or packet containing part of a message, is sent into the LAN and can be heard all over it. The signal rapidly dies away once the transmission has ceased. In a broadcast system, all the users are capable of hearing everything transmitted to them, to another user, or to everybody. When two packets of data are broadcast at the same time, they are said to have collided, and each packet is damaged, $i e$, data are corrupted. A node wishing to transmit listens to the medium (carrier sense) and performs the following: (1) if the medium is idle, then it starts transmitting data; (2) if the medium is busy, then it listens until the channel is sensed to be idle (carrier sense) and subsequently starts transmitting; and (3) if a collision (two nodes start at the same time) is detected, it waits a random amount of time and then repeats steps 1 through 3 . The use of steps 1 through 3 is called a carrier sense multiple access (CSMA) bus. CSMA with collision detection (CSMA/ CD) uses a slight modification to step 3 . If a collision is detected during transmission, it immediately ceases transmitting the frame of data and instead transmits a brief jamming 
signal to ensure that all stations know that there has been a collision. The amount of time it takes to detect a collision is twice the propagation delay at the network. After transmitting the jamming signal, it waits a random amount of time and then transmits again using the CSMA/ $\mathrm{CD}$ access control protocol (ie, Ethernet).

Ring access techniques are accomplished through a repeater placed at each node. Repeater $X$ receives the data packet passed to it by repeater W. Repeater X makes the packet available to the devices attached to node $X$. It then allows all devices at node $X$ to transmit a data packet by transmitting the packet to the next repeater, "Y." A special ring access technique, called token passing, passes a specific bit sequence, called a token, from node to node around the LAN. A node can transmit only when a token is passed to it. Only when its communication is complete does the node pass the token again. The advantages of the token ring access method are: no packet collisions are possible; utilization rate of the $\mathrm{LAN}$ is uniform; and hardware implementation is simple. Token ring access methods may also be used on broadcastbus topology by allowing a node to transmit only when it possesses the token.

The choice of an access method depends upon the LAN application. For data packets consisting of text (Figs 1 and 2), it is best to use a star topology and a token ring access method. This provides good throughput at a reasonable cost. For LAN applications where the data records are long, eg, image management (Fig 3), the best choice depends upon the extent of the department-wide coverage. For an image management LAN in some sections of a department, the broadcast bus with CSMA/CD or token access might be best. A department-wide LAN image management network will probably require a tree topology with fiber optic cables. It is likely that departments will slowly enlarge LAN image management systems by implementing the network in the department section by section. It will be necessary to bridge together the image management of LAN and the patient data LAN.

\section{PREDICTING LAN PERFORMANCE}

The performance of LAN protocols has been extensively studied. ${ }^{27,30-32}$ Careful analysis of the performance of a LAN is difficult but necessary to ensure that promised capabilities are realizable. Three measures often used are: (1) the throughput of the LAN, denoted by "S," ie, the data transfer rate between nodes, of ten called the carried load; (2) the utilization of the LAN medium, denoted by " $\mathrm{U}$," ie, the fraction of total capacity being used; and (3) the delay time, denoted by " $\mathrm{D}$," between initiating transmission and the completion of successful transmission. The parameter $S$ is often normalized and expressed as a fraction of the capacity of the LAN.

The performance of a LAN is the result of the interaction of the four categories discussed and other major factors including the number and type of nodes, their activity, and the amount and character of data on the network. Whether the messages are short (small packets) or long (large packets), they can have major impact on LAN performance which will vary with different LAN configurations. Small packet sizes are common in office automation. Radiology images require large packet sizes. The remainder of this section on performance is a more technical discussion. The use of queueing analysis provides the stochastic tools needed to analyze the performance of a proposed LAN. ${ }^{32}$ However, a simple technique is often used to estimate the upper and lower performance bounds of the LAN protocols. ${ }^{27}$ The assumption is to ignore the medium access control protocol and to know or estimate the following mean times: (1) $T_{I}$, the mean time that a node is idle between transmission attempts; (2) $T_{M}$, the mean time needed to transmit a message once access to the medium is acquired; (3) $T_{D}$, the mean delay from the time a node has a packet to transmit until transmission is completed; and (4) S, the mean total throughput on the network, ie, messages per unit time.

Assume that there are $\mathbf{N}$ active nodes on the network, each identical with the same transmission requirements. An upper bound is found for the total throughput, $\bar{S}$, by assuming each node is in either an "idle" state $\left(T_{I}\right)$ or in a "transmission" state $\left(T_{M}\right)$. The mean throughput for each station is $S=1 /\left(T_{I}+T_{M}\right)$. If no queues occur, $i e$, no node is waiting for another node, then the maximum possible throughput will be $\bar{S}=\mathrm{N} /$ $\left(T_{I}+T_{M}\right)$. Figure 5 illustrates the upper bound on the mean throughput, $\bar{S}$, as a function of $N$. However, as more nodes become active, we soon 


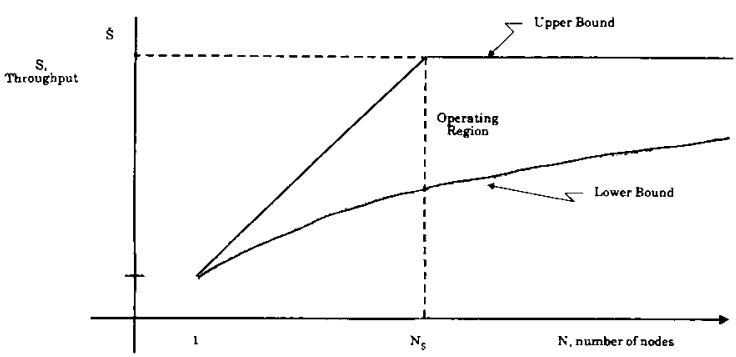

Fig 5. The upper and lower bound on a LAN mean throughput as a function of the number of active nodes, $N$.

approach the saturation value of $\mathrm{N}=\mathrm{N}_{\mathrm{S}}$. At this value of $N$, we no longer have any idle time remaining. The break point occurs when $\mathrm{N}_{\mathrm{S}}$ / $\left(T_{I}+T_{M}\right)=1 / T_{M}$ or $N_{S}=\left(T_{I}+T_{M}\right) /\left(T_{M}\right)$. This simple scheme permits us to size a LAN protocol for upper bounds on the throughput.

A lower bound on mean throughput as a function of $\mathrm{N}$ can be established by assuming that all nodes have a message to transmit simultaneously, then the lower bound for the mean throughput, $\underline{\mathrm{S}}$, would be given by $\underline{\mathrm{S}} \geq \mathrm{N} /$ $\left(T_{I}+N T_{M}\right)$. This is so because we are assuming that each node desiring to transmit a message will have to wait until all other nodes have transmitted. The lower bound, S, is shown in Fig 5 . The actual value of the mean throughput, $S$, will reside somewhere between the upper and lower bounds.

The above method provides a simple model for estimating the mean throughput of a LAN. Improved estimates can be calculated by replacing $T_{M}$ with $T_{\text {sys }}$, where $T_{\text {sys }}$ is the time required to transmit a message plus the overhead time per packet. More accurate estimates can also be attained by considering the medium access protocol. This type of analysis has been carried out for the protocols of CSMA/CD, token bus, and token ring. ${ }^{27,32}$

\section{LAN STANDARDS}

The communication industry long ago learned that standards are required to govern the physical, electrical, and communication protocols for transmitting data. ${ }^{33}$ This lesson was forcefully taught since marketed communication equipment had to interface to that of other manufacturers. The computer industry was slower to accept the need for standards since each manufacturer wanted to provide all needed equipment. However, the growing number of manufacturers of computers, computer devices, and distributed networks has forced the acceptance of standards. A standard is a published set of rules or requirements concerning the terms, components, materials, performance, and operations in describing products, services, or systems. ${ }^{33}$ A number of standards have helped establish the LAN industry.

The International Organization for Standardization (ISO) was founded in 1946 and has issued over 5,000 standards on a broad range of subjects. Technical Committee 97, the information processing system of ISO, published in 1984 the Open Systems Interconnection (OSI) model of a computer communications architecture. The OSI model is accepted and is followed by all LAN manufacturers. The structured set of protocols of the OSI model are layered as shown in Fig 6. The purpose of the OSI model is to devise protocols to perform the functions of each layer. The communication protocols are partitioned into a vertical set of layers, and each layer provides services to the next higher layer. The lowest layer, the physical layer, specifies the electrical and procedural standards for accessing the network. The data link layer provides standards for the reliable transfer of user data over the data link. The network layer determines the most appropriate network configuration for the function being serviced, eg, dial up line or packet switching. The transport layer controls end-toend functions such as routing, error recovery, and

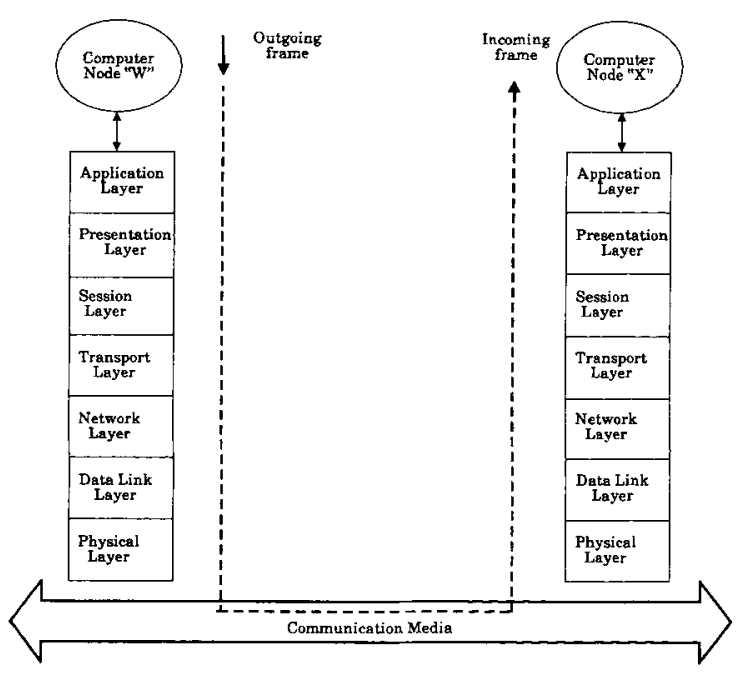

Fig 6. The seven layers of the Open Systems Interconnection (OSI) model. 
message segmentation. A session layer ensures the availability of resources such as file storage and processor time. The presentation layer includes encoding, formatting, interruption and termination. The application layer communicates directly with the user's program or services. A LAN uses the lowest two layers plus portions of the third layer of the OSI model.

In 1980, the Institute of Electrical and Electronics Engineers (IEEE) established the IEEE 802 Local Area Network Committee to standardize the means of connecting digital computer equipment and peripherals to networks. In 1983, the IEEE standards committee board approved standard 802.3 , the CSMA/CD protocol on a bus topology and standard 802.4, the token passing protocol on a bus topology. The 802.2 standard is a logical link control. The 802.5 standard, which details token passing protocol on a ring topology, has been approved. The 802.1 document describes all the 802 standards and their relationship to the ISO Open Systems Interconnection Reference Model. All the IEEE 802 standards are supported by the ISO.

In 1985, a committee of the American College of Radiology (ACR) and the National Electrical Manufacturers Association (NEMA) published the ACR-NEMA Standard 300-1985, "Digital Imaging and Communications." ${ }^{34}$ This standard specifies the hardware interface, software commands, and data formats for communication across an interface between an imaging device and a network interface unit. Additional standards are under development by the ACRNEMA committee, including digital data formats, data compression, and LANs for image management.

Other standard-making organizations influence the LAN standards. The International Telegraph and Telephone Consultative Committee (CCITT) provides standards dealing with the telegraph and telephone. CCITT standards include data communications, telematic services, and integrated services digital networks. The American National Standards Institute is a clearing house for standards in the United States.

\section{DISCUSSION}

The declining costs and widespread applications of LANs make them an ideal extension of communication technology for use in radiology departments. Radiology departments now use portions of LAN technology in computer, office, and information management systems. The implementation of a LAN, using personal computers and file servers, offers an attractive alternative to a system consisting of a large computer with dedicated terminals. LANs are being studied and developed for the department-wide management of patient images and consultation data. The increasing demand for wide-spread applications of LANs ensures that radiology will benefit from continued cost reductions and improved capabilities.

The technology of LANs is rapidly changing. Each month brings announcements of technology improvements and new manufacturers. The half-life of LAN technology is shorter than that of imaging systems in the department. If a radiology department selects a LAN manufacturer, what assurances are there that the manufacturer will be in business the following year? The protection afforded by the OSI interconnections standard ensures that any manufacturers' equipment can be substituted. The best protection is to require department staff members to become knowledgeable in LAN technology and its uses.

Software remains the most expensive and difficult aspect of using and implementing a LAN. Integrating software packages into an information or image management LAN is difficult to accomplish but the power and advantages offered by LANs will undoubtedly lead to their increased implementation in radiology.

\section{REFERENCES}

1. Martin J: Telecommunications and the Computer (ed 3). Englewood Cliffs, NJ, Prentice-Hall, 1976

2. Martin J: Computer Networks and Distribution Processing. Englewood Cliffs, NJ, Prentice-Hall, 1981

3. Tanenbaum AS: Computer Networks. Englewood Cliffs, NJ, Prentice-Hall, 1981
4. Stallings W: Tutorial: Computer Communications: Architectures, Protocols and Standards. Silver Springs, MD, Institute of Electrical and Electronics Engineers, Computer Society Press, 1985

5. Gee KCE: Introduction to Local Area Computer Networks. New York, Wiley, 1983 
6. Stallings W: Local Networks (ed 2). New York, Macmillan, 1987

7. Freeman HA, Thurber KJ: Local Network Equipment. Silver Springs, MD, Institute of Electrical and Electronics Engineers, Computer Society Press, 1985

8. Stallings W: Tutorial: Local Network Technology, (ed 2). Silver Springs, MD, Institute of Electrical and Electronics Engineers, Computer Society Press, 1985

9. Kümmerle K, Tobagi FA, Limb JO, (eds): Advances in Local Area Networks. New York, Institute of Electrical and Electronics Engineers Press, 1987

10. Champine GA: Distributed Computer Systems Impact on Management, Design, and Analysis. Amsterdam, North-Holland, 1980

11. Gupta A, (ed): Advanced Microprocessors, II. New York, Institute of Electrical and Electronics Engineers Press, 1987

12. Taub H, Schilling DL: Principles of Communication Systems (ed 2). New York, McGraw-Hill, 1986

13. Kurosa JF, Schwartz M, Yemini Y: Multiple-access protocols and time-constrained communication. Association of Computer Machinery (ACM) Computing Surveys 16:4370,1984

14. Clark DD, Pogran KT, Roed DP: An introduction to local area networks. Proceedings Institute of Electrical and Electronics Engineers 66:1497-1517, 1978

15. Metcalfe RM, Boggs DR: Ethernet: Distributed packet switching for local computer networks. Commun ACM 19:395-403, 1976

16. Shoch JF, Dalal YK, Redell DD, et al: Evolution of the ethernet local computer network. Computer 5:10-27, 1982

17. Thornton JE, Christensen GS: Hyperchannel network links. Computer 16:50-54, 1983

18. Tropper C: Local Computer Network Technologies. San Diego, Academic, 1981

19. Freeman RL: Telecommunication Transmission Handbook, (ed 2). New York, Wiley, 1981
20. Baker DG: Local-Area Networks With Fiber-Optic Applications. Englewood Cliffs, NJ, Prentice-Hall, 1986

21. Bennett WR, Davey JR: Data Transmission. New York, McGraw-Hill, 1965

22. Lathi BP: Modern Digital and Analog Communication Systems. New York, Holt, Rinehart, and Winston, 1983

23. Alisouskas VF, Tomasi W: Digital and Data Communications. Englewood Cliffs, NJ, Prentice-Hall, 1985

24. Li T: Structures, parameters, and transmission properties of optical fibers. Proceedings Institute of Electrical and Electronics Engineers 68:1175-1180, 1980

25. Bergh AA, Copeland JA: Optical sources for fiber transmission systems. Proceedings Institute of Electrical and Electronics Engineers 68:1240-1247, 1980

26. Smith RG: Photodetectors for fiber transmission systems. Proceedings Institute of Electrical and Electronics Engineers 68:1247-1253, 1980

27. Hammond JL, O'Reilly PJD: Performance Analysis of Local Computer Networks. Reading, MA, AddisonWesley, 1986

28. Abramson N: Information Theory and Coding. New York, McGraw-Hill, 1963

29. Green PE Jr (ed): Computer Network Architectures and Protocols. New York, Plenum, 1982

30. Folts HC, des Jardins R (eds): Special Issue: Open systems interconnection (OSI)-Standard architecture and protocols. Proceedings Institute of Electrical and Electronics Engineers 71:12, 1983

31. Graham GS (ed): Special Issue: Queueing network models of computer system performance. ACM Computing Surveys 10:3, 1978

32. Stuck BW, Arthurs E: A computer and communications network performance analysis primer. Englewood Cliffs, NJ, Prentice-Hall, 1985

33. Stallings $W$ : Handbook of Computer-Communications Standards (vol 1). New York, Macmillan, 1987

34. Wang Y, Best DE, Hoffman JE, et al: ACR-NEMA digital imaging and communications standards: Minimum requirements. Radiology 166:529-532, 1988 